\title{
Las implicaciones místicas-metafísicas, entre la consciencia y el lenguaje."
}

The mystical-metaphysical implications, between

consciousness and language.

\author{
Por: Pereyra Nouveliere, Santiago Agustin * \\ Universidad Nacional de San Juan (UNSJ) \\ E-mail: nouvelieresanty@gmail.com
}

Fecha de recepción: 31/03/2021

Fecha de aprobación: 25/04/2021

DOI: http://dx.doi.org/10.30972/ach.065605

\section{Resumen}

Dentro de este manuscrito, se encontrará una reflexión filosófica a partir de la siguiente gran preocupación. ¿Cómo se manifestaría en el paradigma místicometafísico la pareja conceptual consciencia-lenguaje, como una instancia epistemológica, entiéndase como configuradora de conocimiento? Para ello se manifestará la relación de las implicaciones místicas-metafísicas, aclarando en qué sentido se tomara cada uno de estos elementos. También se trabajará sobre el concepto de consciencia y el concepto de lenguaje, a su vez se establecerá que relación implícita existe entre ellos. Aclarando la vinculación de lo místico-metafísico, con la consciencia y el lenguaje. Para justificar esta hipótesis, que existe la relación antes mencionada, se elaborara un pasaje por

\footnotetext{
* El presente trabajo surge en el marco del proyecto de investigación "El problema de la conciencia en las ciencias contemporáneas: nuevas líneas de investigación”. Resolución N021/18-CS-UNSJ.

* Estudiante avanzado de la licenciatura en Filosofía, de la Facultad de Filosofía, Humanidades y Arte (FFHA). Actualmente trabajando en el Instituto de Investigación de Filosofía (IDEF), dentro del proyecto de investigación mencionado, en la nota al pie de página anterior. También es becario CICITCA, desempeñando sus funciones en el mismo instituto. $\mathrm{Y}$ columnista en RHI (Revista Horizonte Independiente).
} 
varios pensadores sobre la temática. Deteniéndome, con un poco de profundidad, en la filosofía de Ludwig Wittgenstein.

Palabras claves consciencia; lenguaje; mística; metafísica; Wittgenstein.

\begin{abstract}
Within this manuscript, you will find a philosophical reflection from the following great concern. How would the conscience-language conceptual couple manifest itself in the mystical-metaphysical paradigm, as an epistemological instance, understood as a configurator of knowledge? For this, the relationship of the mystical-metaphysical implications will be manifested, clarifying in what sense each of these elements will be taken. We will also work on the concept of consciousness and the concept of language, in turn it will be established what implicit relationship exists between them. Clarifying the link between the mystical-metaphysical, with consciousness and language. To justify this hypothesis, that the aforementioned relationship exists, a passage by several thinkers on the subject will be elaborated. Stopping, with a little depth, in the philosophy of Ludwig Wittgenstein.
\end{abstract}

Key words: consciousness; language; mysticism; metaphysics; Wittgenstein.

Cómo citar este artículo:

APA: Pereyra Nouveliere, S. A. (2021) Las implicaciones místicas-metafísicas, entre la consciencia y el lenguaje. Acheronta, № 6, 150-164. Recuperado de: (agregar dirección web) 
"El conflicto se vuelve insoportable; la exigencia amenaza ahora en convertirse en algo vacío". (Wittgenstein, 2015,109)

El propósito de este ensayo es invitarnos a pensar las posibles relaciones e implicaciones místicos ${ }^{1}$-metafísicos entre la consciencia y el lenguaje. Siendo uno de los objetivos indagar las categorías conceptuales, las cuales están mencionadas en el título, serán sujeto de: reflexiones filosóficas, aclaraciones terminológicas, el sentido en el que van a ser usadas, un rastreo genealógico para denotar las diferentes acepciones que adquieren y han adquirido estos términos en diversos pensadores.

Desde este abordaje surgieron ciertas preguntas posibles como: ¿De qué manera existiría la relación consciencia y lenguaje en el pensamiento filosófico? ¿Cuáles serían los posibles limites en cuanto a la implicaciones lenguajeconsciencia, consciencia-lenguaje? ¿Indagar también si estos conceptos existen o comparten el mismo plano y en qué sentido se relacionarían y habilitarían otros modos de habitar el mundo? Siguiendo estas inquietudes nos aproximamos a otro cuestionamiento, para comenzar a pensar: ¿Cómo se manifestaría en el paradigma místico-metafísico la pareja conceptual consciencia-lenguaje, como una instancia epistemológica, entiéndase como configuradora de conocimiento? Estas indagaciones pueden llegar a tener unas respuestas o permanecer abiertas, para repreguntarnos constantemente y proseguir esta búsqueda, cuando hablo de respuestas también concibo como repuesta valida el silencio o el vacío como límite del lenguaje.

Comenzaré a realizar el análisis genealógico ${ }^{2}$ de la categoría conceptual consciencia. Ella deviene primeramente del verbo griego $\psi u ́ x \omega$, psycho, significa "aire frío». A partir de este verbo se forma el sustantivo $\psi$ uxń, que alude en un

\footnotetext{
${ }^{1}$ Esta palabra "[...] deviene del verbo griego myo, que significa cerrar, clausurar [...]" (Ciner, 2010,13). 2Para Jean Beaufret consiste en: "[...] remontarse [o descender] a las fuentes [...]" (Ferrater, 1964, 746).
} 
primer momento al soplo, hálito o aliento que exhala al morir el ser humano. Por esto termina teniendo repercusiones con el significado de vida. Este verbo se tomará como sinónimo de conciencia en el psicoanálisis (SigmundFreud), de ahí deviene el nombre de su disciplina 'psicología'.

Dicha palabra griega derivara en el latín "conscientia" (Ferrater, 1964,323), y de aquí devendrá en la palabra que utilizamos en español conciencia o consciencia. Tomare ciertas concepciones y definiciones sobre este vocablo para visibilizar cuales van a ser las implicaciones, del concepto conciencia, que trabajare en relación al lenguaje. Ya que ambos conceptos, mencionados anteriormente, poseen una gran variedad de significaciones pero me sería imposible abordarlas todas y no lograría el objetivo de demostrar se muestra esta relación.

Una vez aclarado esto comenzare con las definiciones de consciencia y luego pasare a las de lenguaje, el primer autor que pondré para definir el primer término será Ferrater Mora José quien estudia las dos repercusiones que esta palabra toma dentro del español; de las cuales solo me interesara trabajar la primera $^{3}$, donde la describe de la siguiente manera:

“[...] percatación o reconocimiento de algo, sea de algo exterior, como un objeto, una cualidad, una situación, etc., sea de algo interior, como las modificaciones experimentadas por el propio yo". (ibíd.)

Otra noción sobre la conciencia que tomare es del autor Henri Ey, asemeja consciencia con ser consiente, y al segundo la define como:

"Ser consciente es vivir la particularidad de su propia experiencia, transponiéndola en la universalidad de su saber [...] La organización misma

\footnotetext{
${ }^{3}$ Ya que la segunda concepción, la retoma desde las características de una conciencia moral, no siendo este nuestra prioridad para analizar.
} 
de la vida de relación que ata al sujeto a los demás y a su mundo". (Ey, $1967,11)$

Otra definición de consciencia es la del autor Vygotski, la cual define como: “[...] 'la conciencia', es decir, por recuperar el valor de la mediatización interna entre estímulo y respuesta" (Vygotski, 1995, 13).

Por lo que se puede apreciar, estas diferentes aproximaciones poseen ciertas características similares al definir la conciencia, de hecho la segunda concepción también la podemos encontrar en el diccionario de filosofía:

"Dentro de la conciencia como "ser consciente" pueden distinguirse tres elementos: el objeto de que la conciencia es consciente, la conciencia del objeto, y la conciencia de sí mismo". (Ferrater, 1964, 325)

Claro que más adelante proseguirá que aunque existe tal división los tres están conectados continuamente. Vale aclarar que estas dos corrientes o modos de entender la consciencia, las cuales se expresan en la ambigüedad que toma la palabra, que aparecen en los autores citados con anterioridad, a saber: primero la corriente del ser consciente en relación a un objeto exterior, y segundo la conciencia con el yo interior ${ }^{4}$. Siendo primero la consciencia del yo, y la segunda la conciencia moral. Esta salvedad es de suma importancia de rescatar, pero a lo largo de este ensayo cuando hable de conciencia implicará ambas corrientes, porque bajo mi punto de vista uno no necesariamente excluye al otro.

\footnotetext{
4 En un comienzo entendido a este yo interior bajo la dimensión ontológica, a su vez también comprendida hoy en día bajo la acepción de las diversas escuelas psicológicas.
} 
Nótese aquí que en este apartado se habló en una dicotomía de palabras para utilizar conciencia/consciencia, dicha diferenciación es totalmente necesaria para no caer en errores por las costumbre de nuestra habla, pues aunque son similares tienen repercusiones divergentes tanto en lo lingüístico (esta diferencia también se puede notar en el diccionario de la RAE) como en lo epistémico. Constituyendo la primera palabra el significado de una conciencia moral o de actos morales, la cual como se dijo anteriormente no analizare en este trabajo ya que no es parte de mi objetivo. Mientras que la segunda acepción como consciencia es la que anteriormente se citó pluralidades de modos para comprenderlo teniendo semejantes características, siendo está a la que me referirá de ahora en más cuando hable sobre consciencia.

Ya finalizada la exposición del primer término, proseguiré a realizar la misma labor sobre el segundo concepto, el lenguaje. Realizando el mismo método como en la primera palabra de este artículo, voy a proseguir primeramente con el origen etimológico de la palabra lengua, el cual proviene del latín: “[...] lingua, órgano humano para comer y pronunciar, lenguaje manera de hablar. Derivado lenguado provisto de lengua [...]" (Corominas, 1987, 357)

Para Saussure, los sonidos producidos para comunicarse no serían "[...] más que el instrumento del pensamiento y no existe por sí mismo [...]" (Saussure, $2015,56)$. Este pensador tiene un modo particular de entender al lenguaje que me parece crucial exponerlo, ya que será una de las primeras definiciones formales para poder concebirlo:

“[...] el lenguaje es multiforme y heteróclito; a caballo en diferentes dominios, a la vez físicos, fisiológicos y psíquicos, pertenece además al dominio individual y al dominio social; no se deja clasificar en ninguna de las categorías de los hechos humanos, porque no se sabe cómo desembrollar su unidad". (Saussure,2015, 57) 
Hay una gran variedad en el modo de entender al lenguaje, una de estas que contiene una mirada reduccionista del lenguaje como resultado de un substrato cerebral, uno de los que sostienen este argumento es el psicólogo Pavlov: “[...] el lenguaje explicado en término de la actividad cerebral reflejada" (Vygotski, $1995,9)$.

Otro modo de interpretar el lenguaje, es el de Herder, concibiéndolo del siguiente modo: "[...] el lenguaje no es solo el instrumento, sino también la tesorería, y la forma del pensamiento" [....] "no pensamos solo en un lenguaje determinado, sino a través de ese lenguaje" (Schaff, 1967, 18).

Aunque claro está que el preguntarse por el lenguaje es un preguntarse de antaño, imposible abordarlo en este trabajo, esto se puede observar claramente en la siguiente cita: "La pregunta filosófica por el origen y por la naturaleza del lenguaje - ha escrito Cassirer - es en el fondo tan antigua como la pregunta por la Naturaleza y por él origen del ser" (Ferrater, 1964, 31).

Bastara con estas definiciones para demostrar la divergencia que puede tener el concepto como bien dice Isidoro Rodriguera: "Su pensar pertenece a su lenguaje y su lenguaje pertenece a su pensar" (Wittgenstein, 2015, XXX), por lo que habrá tantos modos de entender y hablar del lenguaje con la cantidad de personas que piensen esta temática, es decir infinita. Incluso en este ensayo daré una interpretación propia entendiéndola como algo innato al hombre que va más allá del habla, siendo este solo una manifestación del primero aunque no la única.

El pensador que se analizará a continuación, una vez ya aclarado ambos conceptos esenciales para esta labor consciencia-lenguaje, es a Ludwig Wittgenstein un pensador profundo que marca una gran ruptura en el modo de filosofar para nuestro tiempos. Dicho pensador posee una ruptura interna en su modo de pensar, por lo que normalmente al estudiarlo se lo divide como dos 
autores separados, valga la salvedad que no por esto su pensar es radicalmente distinto ni muchos menos ya que hay determinadas problemáticas-incógnitas que nunca se van de su escritura así como también conceptos que permanecen como por ejemplo el silencio o el lenguaje; lo que si se modificara es en la perspectiva en que se los observa y analiza, en otras palabras las implicacionesrepercusiones que investirán los conceptos que trabaja, resumido en una palabra su método de trabajo. Dentro de la cantidad de libros que escribió se pueden destacar dos obras pilares, fundamentales para distinguir su interno giro copernicano, en el primer Wittgenstein podemos encontrar el Tratactus lógico-philosophicus (publicada en 1921) y dentro del segundo están los ensayos filosóficos o también llamado investigaciones filosóficas (obra publicada posmortem, en el año 1953 e.c. ${ }^{5}$ ).

Al leer y releer este autor me surgieron grandes incógnitas, con respecto a este tema, algunas de ellas son: ¿Cómo se manifiestan en Wittgenstein las nociones de consciencia y lenguaje? ¿Qué implicaciones posee esta relación para Wittgenstein? Entre otras preguntas si bien podría hacérselas a las dos caras de este pensador, mirando al primero y al segundo como dos caras de la misma moneda, en otras palabras dos posturas de la misma persona. Pero me parece más interesante observarlo en el segundo, aunque no por ello voy a dejar totalmente de lado el primero, ya que bajo mi perspectiva el segundo y definitivo método que adopta resulta una fuente más potable para este trabajo.

Hasta aquí he realizado algunas preguntas, sin embargo no he formulado la gran pregunta, a saber ¿Qué es una pregunta? La cual también se plantea el mismísimo Wittgenstein, realzando ciertas hipótesis del planteo: “¿Es la constatación de que no sé esto y aquello o la constatación de que quisiera que el otro me dijera...? ¿O es la descripción de mi estado mental de

\footnotetext{
5 Iniciales cuyo significado es "era común", para distinguir el ordenamiento de los hechos en los siglos occidentales pero respetando y adhiriéndome al discurso académico laico, al no tener que citar la figura de cristo como modelo universal de referencia histórica.
} 
Abril 2021.

ISSN 2344-9934

incertidumbre?" (Wittgenstein, 2015, 39). Cuya segunda formulación, que realiza para contestar una pregunta respondiendo por otra, es la más provechosa según las preguntas que se realizan a lo largo de todo este ensayo.

Este trabajo del repensarlo cumple en cierta medida con los deseos de nuestro filósofo austriaco, ya sale escrito en el prologo de sus investigaciones: "No quisiera con mi escrito ahorrarles a otros el pensar, sino, si fuera posible, estimular a alguien tener pensamientos propios" (Wittgenstein, 2015, 15).

Un escritor anteriormente mencionado, Isidoro Reguera, realiza un estudio introductorio profundo y basto hacia Wittgenstein por lo cual este ensayo estaría incompleto sin mencionar algunas clarificaciones destacables. Siendo uno de los aportes suyos, el cómo escribió nuestro pensador, cuestión fundamental porque su modo de escribir es radicalmente opuesto al típico modo de pensar y escribir de un pensador sistemático. Esta situación lo plantea de una manera directa la siguiente cita:

“Es verdad que su lenguaje (lo que podría explicar de algún modo esa triste herencia) era un tanto entrecortado, más que aforísticos" [...] "no escribió libros sino notas, trozos de papel que guardaba en cajas de zapatos, que pegaba unos con otros, recortaba, volvía a pegar y recortar de otro modo, buscando siempre cierto orden, que solo consiguió en el caso de las investigaciones" (Wittgenstein, 2015, XXXIII).

Por ello es más complicado estudiar la segunda etapa del pensador, pues a veces carece de sentido el paso de un parágrafo a otro, aunque siempre hay un hilo conductor (no siempre a simple vista) que une sus fragmentos dentro de los ensayos. Una interesante diferencia que se hace notar en su proceso filosófico es la siguiente: 
“Antes para conocer lenguaje y mundo bastaba mirarse - lógicamente- a uno mismo, ahora para conocerse a sí mismo hay que mirar - con sentido común- al lenguaje y al mundo" (Wittgenstein, 2015, LIX).

Profundizando lo denotado en esta cita, en el tratactus Wittgenstein busca un lenguaje universal a través de la utilización de la lógica, por lo tanto ambos conceptos estarían en uno mismo, el problema sería la exteriorización en el lenguaje. Aquí surge una gran incógnita ¿Existe el límite de la consciencia? 0 ¿Hay cosas de la consciencia que no pueden ser expresadas? Este planteo lo veo plasmado en estas palabras que rezan:

"[...] la conciencia de ella - que también pertenece a esa condición, desde la que no se puede decir más que así somos, es decir, admitir su hecho, el de los radicales limites específicos humanos- indecible, inexplicable, no justificable ya, mística humana pues. [...] porque hace falta paz en el pensar de vez en cuando, descansar del circulo de encierro, porque no somos pensamientos (no somos Dios ni Espíritu Absoluto)" (Wittgenstein, 2015, XLIII).

Entonces existe un límite en Wittgenstein sobre la consciencia (según el especialista Reguera), y esto no es poca cosa de hecho ya es bastante para empezar sabemos que existe un límite en nuestra consciencia, pero más que eso somos conscientes nosotros de este límite, cosa muy distinta a afirmar que existe un límite inconsciente a nuestra consciencia. Esto esta expresado de un modo más amplio en las palabras de Reguera:

"Consciencia de límites, en cualquier caso. Es consciencia es algo, el método no es un camino vacío, el método o camino de autoinmolación de 
la filosofía es un despliegue conciencial esplendido, más que apoteósico, al revés: un ritual hominal, en el que fantasmas del pensar se rinden al animal parlante, se inmolan dioses en honor de hombres, la razón es víctima del sentido común, todo ello en una pira final de Alejandría" (Wittgenstein, 2015, LXVI).

Ahora bien estas fronteras de la consciencia están en ambos Wittgenstein pero no bajo del mismo modo, pues en ambos algo hay que callar, en otras palabras existe el silencio en todo su pensamiento. Bien podría verse en estos versos: "O sea, que los dos habrían de callar de algo y habrían callar de todo, o del todo" (Wittgenstein, 2015, XLVII).

Pero el modo en que se manifiesta este silencio es interesante, pues en el Tratactus bien lo expresa bajo el siguiente modo: "De lo que no se puede hablar mejor callar" (Wittgenstein, 2015, 137), es decir caer en vacío en el sentido negativo pues es un silencio obligado, como un imperativo categórico, en otras palabras el callar como mandato. Mientras en la segunda etapa de Wittgenstein, es un silencio optativo porque hay un auto reconocimiento de nuestros límites, pero ya no está este mandato universal del callar.

Justamente en este silencio expresado se encuentra lo místico, es lo que no puede expresarse, mirado en un perspectiva negativa para el primero entrelazándolo con otros conceptos místico-metafísico-religioso, al no poder ser enunciado en un lenguaje lógico universal. Pero admitido humildemente y admirado en el segundo, ya que no somos aun lo místico o lo divino, aun no estamos en ese plano. Como el mismo Wittgenstein afirma, dentro de sus investigaciones, en las palabras se encuentran en la cita que encabeza y da inicio a este trabajo: "El conflicto se vuelve insoportable; la exigencia amenaza ahora en convertirse en algo vacío" (Wittgenstein, 2015,109). Este vacío será uno aceptado y buscado, porque en este vacío vamos a poder encontrar la paz 
que solucionara el lado insoportable, es decir las repercusiones negativas de nuestro preguntar, de nuestra búsqueda.

Ese nuevo papel que juega el silencio en Wittgenstein solo es posible cuando cambia su percepción del lenguaje, es decir ya no va a buscar el lenguaje universal perfecto, por lo tanto ¿Cuál es la concepción que posee en su segunda etapa? Sus propias notas en los ensayos expresa: “[...] imaginar un lenguaje significa imaginar una forma de vida [...]" (Wittgenstein, 2015, 31), esta forma de vida sería «aparentemente» totalmente contrario a su primer trabajo sobre el lenguaje, por lo tanto se opondría al lenguaje entendido en el positivismo en definitiva en contra la definición de Saussure. Pues dice que este concepto del lenguaje radica "[...] en una imagen primitiva del modo y manera en que funciona el lenguaje. Pero también puede decirse que es la imagen de un lenguaje más primitivo que el nuestro" (Wittgenstein, 2015, 19).

Una imagen que muestra de su visión sobre el lenguaje, es la siguiente:

\begin{abstract}
“Nuestro lenguaje puede verse como una vieja ciudad: una maraña de callejas y plazas, de viejas y nuevas casas, y de casas con anexos de diversos periodos; y esto rodeado de un conjunto de barrios nuevos con calles rectas y regulares y con casas uniformes" (Wittgenstein, 2015, 29).
\end{abstract}

Cuyo lenguaje funcionaria a través de juegos, en otras palabras según su uso y no lo que dice, pues como se puede ver anteriormente los conceptos del lenguaje no son universales sino que se reúnen por compartir semejanzas y reglas en un determinado juego. Como él mismo lo define: “[...] Ilamare juegos de lenguaje al todo formado por el lenguaje y las acciones con las que está entretejido" (Wittgenstein, 2015, 23).

Anteriormente mencione que solo «aparentemente» se puede afirmar que se opone totalmente a sus primeros pensamientos, esto lo puedo justificar ya 
Abril 2021.

que este filósofo considera a los géneros de oraciones como innumerables así se puede ver en sus palabras:

“[...] innumerables géneros diferentes de empleo de todo lo que llamamos signos, palabras, oraciones. Y esta multiplicidad no es algo fijo, dado una vez por todas; sino que nuevos tipos de lenguaje, nuevos juegos de lenguaje, como podemos decir, nacen y otros envejecen y se olvidan" (Wittgenstein, 2015, 37).

Bajo este argumento de multiplicidad y devenir, se puede admitir también las posturas sobre el lenguaje dentro del Tratactus así como esa línea de pensamientos ya que sería un modo más de concebir el lenguaje a través de uno de sus juegos, en otras palabras seria un genero más. El problema radicaría si se toma este género como único y universal, porque ahí si entraría en conflictos con la teoría de la multiplicidad, es decir que la única afirmación universal sobre el lenguaje es afirmar su multiplicidad.

Por lo trabajado hasta este punto me parece importante expresar este movimiento dialéctico ${ }^{6}$ entre consciencia-lenguaje-silencio, y no solamente consciencia y lenguaje, al menos que se pueda concebir o integrar el silencio al lenguaje. Pero en este caso me parece importante nombrarlos los tres por separados ya que poseen una gran importancia en esta dinámica. Dicho movimiento lo fundamento como una afirmación inicial del ser consciente, una negación que se da en el lenguaje al intentar expresar este ser consciente por el lenguaje para auto conocerse, y una última reacción que desembocaría de la anterior el silencio donde se manifiesta lo místico. Un ser consciente, no solo de

\footnotetext{
${ }^{6}$ Entiéndase como proceso del devenir, tomado desde Hegel con: una afirmación, una negación y una negación de la negación.
} 
su limitación, sino de algo más; por lo que me parece interesante nombrarlo un silencio-místico-metafísico.

Con todo lo anteriormente analizado, que solo conforma un pequeño recorrido la problemática, es decir solo conforma el inicio de esta investigación y no la conclusión de ella. Se podría afirmar que este silencio-místicometafísico, en el cual no habría un ser consciente nuestro, propiamente dicho en el sentido trabajado aquí con Wittgenstein, pero si habría algo más dentro del cual también poseemos una participación. Esto otro podría nombrarse también como alma, lo cual me trae otra incógnita ¿puede ser el alma una parte de la consciencia que no es consciente de sí misma? O también podría pensarse de este modo ¿Después del límite silencioso de consciencia puede estar el alma? O ¿El alma está implicada dentro del silencio que marca el límite de la consciencia? 
Bibliografía:

Grupo interdisciplinario de investigación mente y lenguaje, La Pontificia Universidad Católica de Perú, recuperado de: http://menteylenguaje.pucp.edu.pe/

Carmona C. (2015): "Wittgenstein, La conciencia del límite", Buenos Aires, Bonalletra Alcompas.

Ciner P. (2010): "La experiencia mística, líneas actuales de investigación", San Juan, Facultad de Filosofía, Humanidades y Arte.

Concienciame (2017, 03, 05): "El poder de la mente: Consciencia / Documental Completo", recuperado de: https://www.youtube.com/watch?v=9 29HKDx500

Corominas J. (1987): "Breve diccionario etimológico de la lengua castellana",

\section{España, Gredos.}

Ey H (1967): "La conciencia”, Madrid, España, Gredos.

Ferrater J. (1964): "Diccionario de Filosofía", Tomo I, Buenos Aires, Sudamericana.

Ferrater J. (1964): "Diccionario de Filosofía", Tomo II, Buenos Aires, Sudamericana

Saussure F. (2015): "Curso de lingüística general", Buenos Aires, Losada.

Schaff A. (1967): “Lenguaje y conocimiento”,México, Grijalbo.

Vygotski L. (1995): “Pensamiento y lenguaje”, España, Fausto.

Wittgenstein L. (2015): "Ludwig Wittgenstein I", España, Gredos.

Wittgenstein L. (2015): “Ludwig Wittgenstein II", España, Gredos 